\title{
Zwischen Kooperation und Grenzziehung \\ - Aushandlungen von Sicherheitsbehörden und Akteur*innen Sozialer Arbeit in der Radikalisierungsprävention
}

\author{
Carmen Figlestahler $\cdot$ Katja Schau
}

Eingegangen: 30. September 2019 / Angenommen: 23. März 2020 / Online publiziert: 9. Dezember 2020 (C) Der/die Autor(en) 2020

Zusammenfassung Die Herstellung verschiedener Formen von Sicherheit sowie die Zusammenarbeit mit Sicherheitsbehörden sind in unterschiedlichen Arbeitsfeldern der Sozialen Arbeit relevant. Im vorliegenden Beitrag wird dieser allgemeine Sachverhalt für den speziellen Bereich der indizierten Prävention und Ausstiegsarbeit von demokratiefeindlichem Islamismus betrachtet. Hier spielen Narrative der Abwehr potenzieller Gefahren für die innere Sicherheit eine besondere Rolle und es werden mitunter sehr konkrete Kooperationserwartungen von Sicherheitsbehörden an Soziale Arbeit gestellt. Jedoch gibt es bisher wenige empirisch fundierte Analysen, welche die Auswirkungen dieser vergleichsweise engeren Kooperationen auf die praktische Bearbeitung von demokratiefeindlichem Islamismus bzw. die Aushandlung von Zuständigkeiten untersuchen. Anhand einer qualitativen Fallstudie zum regionalen Kooperationsarrangement untersuchen wir die multiprofessionelle Problembearbeitung von demokratiefeindlichem Islamismus und gehen der Frage nach, wie Informationsweitergaben und Grundprinzipien der Arbeit zwischen sicherheitsbehördlichen und sozialpädagogischen Akteur*innen ausgehandelt werden. Dabei zeigt sich beispielsweise, dass sozialpädagogische Akteur*innen Kooperationsanforderungen der Sicherheitsbehörden nicht einfach übernehmen, sondern durchaus selbstbewusste Grenzen der Zuständigkeiten mit Rückgriff auf grundlegende fachliche Prinzipien ziehen.

Schlüsselwörter Sicherheit · Versicherheitlichung · multiprofessionelle Kooperation · Islamismus · Prävention · Radikalisierungsprävention

Dr. C. Figlestahler $(\bowtie) \cdot$ K. Schau, M.A.

Außenstelle Halle, Deutsches Jugendinstitut, Franckeplatz 1, 06110 Halle, Deutschland

E-Mail: figlestahler@dji.de

K. Schau, M.A.

E-Mail: schau@dji.de 


\title{
Between cooperation and the drawing of boundaries-negotiations of security-related actors and actors of social work in the prevention of radicalisation
}

\begin{abstract}
The fabrication of different forms of "security" as well as cooperation with security-related actors (e.g. police) are relevant issues in different areas of social work. In this article we examine this general matter focusing on the specific field of indicated prevention and exit work of anti-democratic Islamism. Here narratives about the defence of potential threats for domestic security play a major role and security-related actors occasionally make explicit requests for cooperation towards actors of social work. Based on a qualitative case study of a regional cooperation setup we analyse the work on anti-democratic Islamism in a multi-agency setting. We focus on the negotiation of responsibilities between actors of social work and security-related actors. Our findings show for example, that actors of social work do not simply adopt requests for cooperation by security-related actors, instead assertively drawing boundaries referring to the principles of social work.
\end{abstract}

Keywords security $\cdot$ securitisation $\cdot$ multi-agency cooperation · Islamism · prevention $\cdot$ prevention of radicalisation

\section{Einleitung}

Die Herstellung verschiedener Formen von Sicherheit sowie die Zusammenarbeit mit Sicherheitsbehörden sind in unterschiedlichen Arbeitsfeldern der Sozialen Arbeit relevant (z. B. Dollinger et al. 2017; Franzheld 2017). Dabei muss das multiprofessionelle Verhältnis zwischen sozialpädagogischen und sicherheitsbehördlichen Akteur*innen immer wieder ausgehandelt und ausbalanciert werden. Zu klärende Fragen sind dabei beispielsweise, wer mit welchen Zielgruppen arbeiten „darf“ und welche Informationen an wen weitergegeben werden ,müssen“. Im Feld der Präventions- und Ausstiegsarbeit von demokratiefeindlichem Islamismus ${ }^{1}$ spielen Narrative der Abwehr potenzieller Gefahren für die innere Sicherheit eine besondere Rolle. Verbunden mit dem Auftrag an Sicherheitsbehörden, innere Sicherheit herzustellen, werden mitunter konkrete Kooperationserwartungen von Sicherheitsbehörden an Soziale Arbeit gestellt. Angesichts dessen konstatieren einzelne kritische Stimmen Tendenzen einer Versicherheitlichung für dieses spezifische Feld der Sozialen Arbeit, d.h. einer Gewichtsverschiebung zugunsten sicherheitspolitischer Perspektiven (Schuhmacher 2018a; Jukschat und Leimbach 2019). ${ }^{2}$ Jedoch gibt es bisher wenige empirisch fundierte Analysen, welche die Auswirkungen dieser vergleichsweise

\footnotetext{
${ }^{1}$ Über die Bezeichnung des sozialen Problems herrscht in den Fachdebatten kein Konsens. Wir sprechen im Folgenden von „demokratiefeindlichem Islamismus“. Dieser Begriff ist zwar wegen der semantischen Nähe zum Islam im Allgemeinen umstritten, erscheint uns mit der Spezifizierung „,demokratiefeindlich“ im Moment jedoch als geeignete Variante.

2 In Großbritannien werden mögliche Konsequenzen der sicherheitspolitisch gerahmten Extremismusprävention im Rahmen des Programms „Prevent“ seit längerem in der Fachliteratur diskutiert (z. B. Durodie 2016).
} 
engeren Kooperationen auf die praktische Bearbeitung von demokratiefeindlichem Islamismus untersuchen. Gerade handlungsbezogene Fragestellungen, etwa wie sozialpädagogische Akteur*innen mit sicherheitsbehördlichen Anforderungen umgehen und wie sie in Aushandlungsprozessen agieren, wurden bisher kaum vertieft analysiert. Um Fragen möglicher Tendenzen einer Versicherheitlichung in diesem Handlungsfeld empirisch zu fundieren, stellen wir im Folgenden die Befunde einer explorativen, qualitativen Fallstudie zu einem regionalen Bearbeitungsarrangement vor. Wir fokussieren uns dabei auf Aspekte der multiprofessionellen Kooperation: Aus einer interaktionistischen sowie macht- und professionstheoretischen Perspektive gehen wir u. a. der Frage nach, wie Informationsweitergaben und Grundprinzipien der Arbeit zwischen sicherheitsbehördlichen und sozialpädagogischen Akteur*innen im Bereich des demokratiefeindlichen Islamismus ausgehandelt werden. Damit leistet die vorliegende Fallstudie einen Beitrag dazu, die empiriebezogene Forschungslücke zu schließen.

\section{Sozialpädagogische Islamismusprävention als ein Feld mit versicherheitlichten Kooperationsarrangements?}

Die sozialpädagogische Bearbeitung von demokratiefeindlichem Islamismus hat sich in Deutschland seit rund zehn Jahren zu einem eigenen Handlungsfeld entwickelt, das sich vor allem seit ca. 2014 im Zuge der Etablierung verschiedener Angebotsstrukturen erheblich ausgeweitet und diversifiziert hat. Die Durchführung dieser Angebote erfolgt größtenteils durch zivilgesellschaftliche Akteure, die häufig freie Träger der Kinder- und Jugendhilfe sind (Schau et al. 2017). Wir fokussieren uns auf den Bereich der indizierten Prävention bzw. Ausstiegsarbeit. Hier wird mit jungen Menschen, die sich (möglicherweise) in Hinwendungsprozessen zu problematischen Gruppen befinden oder aus demokratiefeindlichen islamistischen Szenen lösen wollen, sozialpädagogisch gearbeitet, i.d.R. im Rahmen von Einzelfallhilfe. ${ }^{3}$ Im Folgenden gehen wir der Frage nach, ob es sich bei der sozialpädagogischen Bearbeitung von demokratiefeindlichem Islamismus um einen Bereich Sozialer Arbeit handelt, der derart von Sicherheitslogiken dominiert wird, dass man von einem versicherheitlichten Handlungsfeld sprechen kann.

\subsection{Tendenzen der Versicherheitlichung}

Im Vergleich zu anderen Feldern Sozialer Arbeit im Allgemeinen aber auch zur bisherigen Bearbeitung von Rechtsextremismus im Besonderen, ist dieses Arbeitsfeld stärker sicherheitspolitisch gerahmt und die Zusammenarbeit mit sicherheitsbehördlichen Akteur*innen, welche einzelne Angebote zum Teil finanzieren und koordinie-

\footnotetext{
3 Die Unterscheidung zwischen indizierter Prävention und Ausstiegsarbeit beruht auf einer analytischen Differenzierung der Zielgruppen, je nach ihren Involvierungsgraden: Während sich Angebote der indizierten Prävention an junge Menschen im Hinwendungsprozess zu problematischen Gruppen bzw. Ideologien richten, umfasst Ausstiegsarbeit demobilisierende und deradikalisierende Arbeit mit bereits szeneinternen, ausstiegswilligen Menschen. In der Praxis verschwimmen eindeutige Grenzziehungen zwischen beiden Bereichen.
} 
ren, spielt eine größere Rolle (Schuhmacher 2018a; Said und Fouad 2018). ${ }^{4}$ Diese Rahmung ist einerseits nicht losgelöst von allgemeinen gesellschaftlichen Entwicklungen zu verstehen, die das Verständnis und die Praxis Sozialer Arbeit prägen. So ist Sicherheit v.a. in den letzten beiden Jahrzehnten zu einem ,Schlüsselkonzept westlicher Gesellschaften geworden“ (Singelnstein und Stolle 2012, S. 122) und die Vorstellung, alle möglichen Risiken und Gefahren kontrollieren bzw. durch Präventionsstrategien verhindern zu können, hat an gesellschaftlicher Prägekraft gewonnen (Groenemeyer 2013; Bröckling 2008). Mit dem Schlagwort der Versicherheitlichung wird dabei ein Prozess bezeichnet, in dem sicherheitspolitische Perspektiven an Gewicht zunehmen und soziale Sachverhalte unter dem Fokus einer potenziellen Sicherheitsrelevanz wahrgenommen werden (Dollinger 2014, S. 299). Andererseits liegt die sicherheitspolitische Rahmung des hier betrachteten Handlungsfeldes auch darin begründet, dass islamistisch motivierte Anschläge in Deutschland seit einigen Jahren als zentrale gesellschaftliche Bedrohung der inneren Sicherheit gelten (Bundesministerium des Innern (BMI) 2017a). ${ }^{5}$ So ist auf politisch-konzeptioneller Ebene eine vergleichsweise enge Kooperation von sicherheitsbehördlichen und sozialpädagogischen Vorgehensweisen zum Ziel des Gesellschaftsschutzes angedacht. Gemeinsam soll u.a. Gewalt, verfassungsfeindlichen Bestrebungen und Gefahren für die demokratische Ordnung entgegengewirkt werden. Im 2017 von der Bundesregierung beschlossenen Nationalen Präventionsprogramm (NPP) gegen islamistischen Extremismus heißt es beispielsweise: „Wir müssen ansetzen, bevor Radikalisierungsprozesse in Terrorgefahr umschlagen. Darum müssen Prävention und Repression Hand in Hand gehen“" (BMI 2017b, S. 1). Damit wird ein direkter Zusammenhang zwischen Prävention und Terrorabwehr hergestellt und ein gemeinsames Vorgehen von (sozialpädagogischer) Prävention und repressiven Maßnahmen von Sicherheitsbehörden als Idealbild festgeschrieben. Schließlich sind, bezogen auf die konkrete Bearbeitungspraxis, gerade in der indizierten Prävention bzw. der Ausstiegsarbeit mögliche Ausreisen in Kampfgebiete des Islamischen Staats (IS) und der Umgang mit Rückkehrenden aus diesen Gebieten mitunter Gegenstand der sozialpädagogischen Arbeit. Da diese Fälle von potenzieller Relevanz für Fragen der inneren Sicherheit sind, liegen sie teilweise auch im Zuständigkeitsbereich sicherheitsbehördlicher Akteure.

Lässt sich aufgrund dieser sicherheitspolitischen Rahmung von einem versicherheitlichten Handlungsfeld sprechen? Aus Perspektive der Praxis stellen zivilgesellschaftliche Organisationen im Handlungsfeld wachsende Kontrollversuche durch Sicherheitsbehörden bzw. eine sicherheitspolitische Schwerpunktsetzung fest (z.B. Handle et al. 2020; Nordbruch 2018; Möller und Neuscheler 2018). Aus wissenschaftlicher Sicht konstatiert Nils Schuhmacher (2018a) mit Blick auf das Handlungsfeld zumindest insoweit entsprechende Tendenzen, als es seines Erachtens ei-

\footnotetext{
4 Die jeweilige Form der Kooperation zwischen Sicherheitsbehörden, Innenministerien, Sozialministerien sowie sozialpädagogischen Akteur*innen unterscheidet sich dabei je nach Bundesland bzw. Förderstruktur.

5 Politische Akteur*innen haben islamistisch begründete Terroranschläge in den USA und Europa in den letzten Jahren immer wieder als Legitimationsgrundlage für die Verschärfung von Gesetzen mit Sicherheitsbezug und zur Ausweitung sicherheitsbehördlicher Befugnisse herangezogen (z. B. Heinrich 2017; Scherr 2014; Baban 2013).
} 
ne Gewichtsverlagerung zugunsten sicherheitspolitischer Perspektiven in pädagogischen Kontexten gebe (Schuhmacher 2018a). Dieser Prozess zeichne sich erstens anhand einer Versicherheitlichung der Präventionsidee ab, wenn sozialpädagogische Arbeit dem übergeordneten Ziel der inneren Sicherheit diene und ihr Erfolg an der Erreichung dieses Ziels festgemacht werde. Unmittelbar damit verbunden ist zweitens die Vorstellung der Messbarkeit von Wirkungen sozialpädagogischen Handelns und der Erwartung, dass Praktiker*innen sich auf die Logik (Risiko-)prognostischer Instrumente einlassen. Drittens benennt er schließlich den Trend zur Etablierung von multiprofessionellen Strukturen, die zunehmend sicherheitspolitisch gerahmt würden (Schuhmacher 2018a, S. $161 \mathrm{ff}$.).

In solchen multiprofessionellen Kooperationen gibt es potenzielle Spannungsfelder zwischen Sicherheitsbehörden und Akteur*innen Sozialer Arbeit, da sie sich in ihren Aufträgen, Zielsetzungen, Arbeitsprinzipien und rechtlichen Rahmenbedingungen grundlegend unterscheiden. So steht z. B. dem Auftrag der Gefahrenabwehr die Aufgabe gegenüber, individuelle Handlungsfähigkeit herzustellen und soziale Teilhabe zu ermöglichen (Möller 2010, S. 17 ff.). ${ }^{6}$ Für die vorliegende Betrachtung ist vor allem die Differenz in Bezug auf Wissen relevant. Während sozialpädagogische Praxis lückenhaftes Wissen über ihre Adressat*innen anerkennt, möchten Sicherheitsbehörden solche Lücken aufgrund ihres Auftrags des gesamtgesellschaftlichen Schutzes tendenziell schließen. Diese Diskrepanz lässt sich in multiprofessionellen Kooperationen nicht einfach durch eine Festlegung der Regeln für Datenaustausch aufheben. Vielmehr sei davon auszugehen, ,dass in einer versicherheitlichten Wissenskultur der Druck steigt, diese Differenz in Richtung der Wissensmaximierung aufzulösen“ (Schuhmacher 2018a, S. 164). Aspekte von gesellschaftlicher Kontrolle sind allerdings auch für Soziale Arbeit seit ihrem Bestehen konstitutiv und sie agiert immer in einem Spannungsfeld von Hilfe und Kontrolle (z. B. Müller 2001; Huber und Schierz 2013, S. 103 ff.). Dabei ist der dezidierte Bezug zu Fragen der Sicherheit und des Schutzes vor Risiken sowie die Zusammenarbeit mit sicherheitsbehördlichen Akteuren in bestimmten Feldern Sozialer Arbeit wie etwa dem Kindesschutz und dem Umgang mit Jugendgewalt besonders ausgeprägt. Auch hier gibt es die Tendenz, sozialpädagogische Akteure vermehrt als Instanzen zu sehen, die im Dienst von Sicherheitsbehörden die Bevölkerung vor Risiken schützen sollen (Dollinger 2014). Verschiedene Analysen zeigen dabei, dass es hier zwar zum Teil eine Verschiebung in Richtung einer angestrebten Dominanz sicherheitspolitischer Perspektiven gibt, es würde aber zu weit führen, pauschal von der Tendenz zur Versicherheitlichung für die Soziale Arbeit im Allgemeinen zu sprechen (vgl. Dollinger 2014; Huber und Schierz 2013). Ähnliches gilt für das Feld der sozialpädagogischen Bearbeitung von demokratiefeindlichem Islamismus: Es lässt sich zwar beobachten, dass Sicherheitslogiken eine große Rolle spielen, doch lässt sich auf Basis einer strukturbezogenen Beschreibung schwer einordnen, wie sich das in der Praxis niederschlägt.

\footnotetext{
6 Kurt Möller bezieht sich in seinen Ausführungen auf die Polizei. In der Bearbeitung von demokratiefeindlichem Islamismus ist daneben auch der Verfassungsschutz ein relevanter sicherheitsbehördlicher Akteur, auf den die genannten Differenzen in ihren allgemeinen Grundzügen ebenfalls zutreffen.
} 


\subsection{Multiprofessionelle Kooperationsarrangements als Aushandlung von Zuständigkeiten}

Wie deutlich wurde, stellt die Etablierung von multiprofessionellen Strukturen der Zusammenarbeit, in denen Sicherheitsbehörden dominieren (wollen), einen wesentlichen Aspekt einer möglichen Versicherheitlichung dar. Dabei ist Sozialer Arbeit generell die Handlungsmaxime eingeschrieben, mit anderen Professionellen und Institutionen zu kooperieren (Merten 2016). Ihre Angebote zielen grundsätzlich darauf ab, Adressat*innen in ihren unterschiedlichen Problem- und Lebenslagen zu unterstützen und ihnen einzelfallorientierte Hilfeangebote zu offerieren. Dafür braucht es auch das Abklären von verschiedenen Handlungsmöglichkeiten sowie das Abstimmen von unterschiedlichen Angeboten und Interventionen. Interaktionistische Perspektiven auf Kooperationen in der Sozialen Arbeit betonen, dass die gemeinsamen Ziele und Handlungsmöglichkeiten verschiedener Professionen im Einzelfall nicht einfach vorliegen, sondern in kommunikativen Prozessen zwischen den Fachkräften ausgehandelt werden (Merten 2016; Schütze 1992). Zentraler Gegenstand der Aushandlung, gerade auch mit anderen Professionellen, sind dabei Fragen der rechtlich verankerten und professionsethisch fundierten (Fall-)Zuständigkeit und Informationsweitergabe an andere beteiligte Professionelle (Silkenbeumer et al. 2017; Franzheld 2017; Abbott 1988).

Vergegenwärtigt man sich Anforderungen in multiprofessionellen Aushandlungsprozessen, dann liegt die macht- und professionstheoretische Perspektive von Andrew Abbott (1988) nahe. So werden die professionseigenen Zuständigkeiten nicht nur kontinuierlich ausgehandelt, sondern auch behauptet und durchgesetzt. Dabei setzt A. Abbott (1988) die Zuständigkeitssicherung mit professionsbezogener Autonomiesicherung gleich. Es manifestiere sich die gesellschaftliche Anerkennung der Leistung zur Problemlösung auch in den inter- und intraprofessionellen Machtkämpfen um Zuständigkeit und professionelle Außengrenzen (auch Franzheld 2017; Abbott 1988). ${ }^{7}$

Auch wenn im hier beschriebenen Überlappungsbereich zwischen Sozialer Arbeit und Sicherheitsbehörden keine grundsätzliche Infragestellung der Bearbeitungsleistung der Sozialen Arbeit erfolgt, so konstatieren Kurt Möller und Florian Neuscheler (2018) doch eine asymmetrische Konstellation zwischen den beiden Professionen. Akteur*innen der Sozialen Arbeit sind stärker von öffentlichen Geldern und Aufmerksamkeiten abhängig und agieren vielfach in befristeten Projekten. In den Aushandlungen der beiden Professionen ist - trotz allem kooperativen Pragmatismus wichtig, das eigene Profil der Sozialen Arbeit fachlich zu schärfen und offensiver nach außen zu präsentieren. So entsteht ein (Ver-)Handlungsspielraum, der einseitige externe Kontrollansprüche und eine ordnungspolitische Inanspruchnahme der Sozialen Arbeit fachlich eindämmen kann (vgl. Huber und Rieker 2015). Dies ermöglicht es auch in asymmetrischen Machtkonstellationen mit Rückgriff auf professionelle

\footnotetext{
7 Thomas Klatetzki (2005) arbeitete heraus, dass Soziale Arbeit in verschiedenen gesellschaftlichen Bereichen fortwährend in ihrer Zuständigkeit und Bearbeitung von sozialen Problemen in Frage gestellt wird. Mit Andrew Abbott (1988) ist die Unterschätzung der sozialpädagogischen Handlungsfähigkeit Ausgangspunkt für einen kontinuierlichen Kampf um professionelle Legitimität.
} 
Selbstverständnisse Grenzen zu ziehen und beispielsweise auf das Spannungsverhältnis von Hilfe und Kontrolle sowie auf die Erteilung des Handlungsmandats zu verweisen.

Zusammenfassend lässt sich feststellen, dass gerade im vergleichsweise jungen Feld der sozialpädagogischen Bearbeitung von demokratiefeindlichem Islamismus die Betrachtung multiprofessioneller Aushandlungen aufschlussreich für die Untersuchung möglicher Tendenzen einer Versicherheitlichung ist. Gibt es z.B. Anzeichen für versicherheitlichte Kooperationsarrangements, in denen sicherheitsbehördliche Perspektiven in der Aushandlung dominant sind und deren Ergebnisse bestimmen? Wie im vorangegangenen Abschnitt beschrieben, sind entsprechende Diagnosen im bisherigen Diskurs eher größere, pauschale Tendenzbeschreibungen, denen es oft an empirischen Untersetzungen fehlt. Aktuelle Evaluationsstudien zu Trägern in der indizierten Prävention und Ausstiegsarbeit verweisen zwar am Rande auf diese Aushandlungskonstellationen und Abgrenzungsanforderungen (Schuhmacher 2018b; Möller und Neuscheler 2018), es fehlen jedoch weiterhin vertiefende empirische Untersuchungen zur konkreten Aushandlungspraxis.

\section{Hintergrund der Studie und methodisches Vorgehen}

Ausgangspunkt für die Erhebung der empirischen Daten, die diesem Beitrag zugrundliegen, war eine regionale Fallstudie im Kontext der wissenschaftlichen Begleitung der Modellprojekte der Radikalisierungsprävention im Bundesprogramm „Demokratie leben!“‘. Dafür wählten wir ein Bundesland aus, in dem die Bearbeitung von demokratiefeindlichem Islamismus ein relevantes Thema ist. Dort gibt es mehrere Städte, die als Hotspots für islamistische Radikalisierungen in Deutschland gelten. Im Frühjahr 2018 führten wir vier leitfadengestützte Interviews mit den zu diesem Zeitpunkt zentralen Akteur*innen der Bearbeitung. Dies sind zum einen zwei zivilgesellschaftliche Projekte, die in unterschiedlichen Städten angesiedelt sind: Projekt Porto $^{8}$ ist bei einem Träger der freien Kinder- und Jugendhilfe angesiedelt und verortet sich selbst in der Sozialen Arbeit. Projekt Prag hat stärker religiöse Bezüge und wird von einem muslimischen Verband getragen. Ein weiterer relevanter Akteur ist eine koordinierende Stelle des Sozialministeriums, sie wird mit dem Kürzel KSM bezeichnet. Schließlich haben wir ein Interview mit einem Vertreter einer Sicherheitsbehörde geführt, die dem Innenministerium unterstellt ist, im Folgenden: SIM.

Der Forschungsprozess war, den Grundprinzipien der Grounded Theory (Strauss 1994) folgend, iterativ ausgerichtet. Die Interviews waren mit dem Ziel der allgemeinen Darstellung des lokalen Bearbeitungs-Arrangements erhoben worden. Um den Fokus der Auswertung zu schärfen, haben wir den Gegenstand der Analyse nach der ersten Durchsicht des Materials konkretisiert und mögliche Versicherheitlichstendenzen in der sozialpädagogischen Bearbeitung von demokratiefeindlichem Islamismus in den Blick genommen. Im nächsten Schritt wurden Schlüsselstellen

\footnotetext{
${ }^{8}$ Die erhobenen Daten wurden anonymisiert bzw. pseudonymisiert, d. h. zum Teil wurden einzelne Angaben gezielt geändert, um eine mögliche Identifikation zu verhindern.
} 
für diese Themen ausgewählt. Die Auswertung orientierte sich an der sozialwissenschaftlichen Hermeneutik. Dabei wurden Selbst- und Fremddeutungen der unterschiedlichen Akteur*innen analysiert, ihre manifesten Erfahrungen (weil-Motive) und damit verknüpften Absichten (um-zu-Motive) interpretiert und nach und nach der interaktionsbezogene Sinn erschlossen (Soeffner 2008, S. $219 \mathrm{ff}$.). Die empirische Basis der vorliegenden Fallstudie ist zwar verhältnismäßig klein, doch geht es den Prämissen der sozialwissenschaftlichen Hermeneutik folgend nicht darum, eine Verallgemeinerung durch die Bearbeitung einer möglichst großen Menge von Daten zu erreichen. Vielmehr wird davon ausgegangen, dass sich das Allgemeine an der Besonderheit exemplarischer Fälle zeigen lässt (Soeffner 2008, S. 158).

Die Interviews wurden jeweils mit einer Person realisiert. Auf dieser Basis lassen sich nicht ohne Weiteres verallgemeinernde Schlussfolgerungen über die gesamte Institution ziehen. Daher haben wir in der Analyse auch auf die subjektive Bezugnahme zur jeweiligen Institution geachtet, in der folgenden empirischen Darstellung differenzieren wir dies nicht weiter aus, weisen aber in einem Fall bewusst darauf hin, dass sich die interviewte Person teilweise von der Handlungslogik der Institution distanziert.

Im Folgenden beschreiben wir zunächst empirisch verdichtet das regionale Bearbeitungsarrangement in Bezug auf die Arbeitsfelder der Akteur*innen sowie die wahrgenommene Relevanz des Problems; dabei zeigen sich grundsätzliche Differenzen, die mit unterschiedlichen Kooperationsinteressen einhergehen (Abschn. 4). Darauf aufbauend stellen wir am Beispiel der Aushandlung von Zuständigkeiten in der Ausstiegsarbeit die Versuche von Sicherheitsbehörden dar, Arbeitsaufträge zu vermitteln und in Reaktion darauf die Grenzziehungen von sozialpädagogischen Akteur*innen (Abschn. 5). Anschließend rekonstruieren wir in einer vertieften Analyse von zwei Schlüsselstellen die unterschiedlichen Positionierungen von zwei Akteur*innen und arbeiten heraus, welche Rolle forcierte Sicherheitsinteressen spielen und inwiefern sicherheitsbezogene Kooperationsinteressen machtvoll durchgesetzt werden können (Abschn. 6).

\section{Arbeitsfelder, Problemdeutungen und Kooperationsinteressen}

Prinzipiell sind alle vier Akteur*innen für die Bearbeitung von demokratiefeindlichem Islamismus zuständig, sie unterscheiden sich jedoch hinsichtlich ihrer konkreten Arbeitsfelder, ihrer Deutungen des sozialen Problems und der Kooperationsinteressen. So fokussiert sich die SIM als sicherheitsbehördlicher Akteur auf die Abwehr von ,gewaltorientiertem Salafismus“ (SIM, Z. 190). Dieses Phänomen stellt aus ihrer Sicht ein drängendes soziales Problem in ihrem Bundesland dar und entsprechend hat die Bearbeitung ihres Erachtens hohe Priorität. Vor dem Hintergrund ihres Auftrags, innere Sicherheit zu gewährleisten, haben sie ein hohes Interesse daran, in umfassender Form mit den sozialpädagogischen Akteur*innen zu kooperieren - nicht zuletzt, da für sie die Notwendigkeit besteht, möglichst weitreichende Informationen zu potenziell sicherheitsrelevanten Fällen zu generieren.

Die KSM teilt die Einschätzung der hohen Dringlichkeit des Problems nicht in gleicher Weise, sondern betont die faktisch höhere Relevanz von Rechtsextremismus. 
Jedoch gebe es mehr (politische) Nach- und Anfragen, auch zur multiprofessionellen Zusammenarbeit, zum Thema demokratiefeindlicher Islamismus. Gleichwohl gebe es ihres Erachtens eine große salafistische Szene vor Ort, sie sehen es jedoch als ihre Aufgabe an, die teils alarmistisch geführten Debatten zu entschärfen und die „Aufgeregtheit" (KSM, Z. 218) aus der Diskussion um adäquate Umgangsweisen zu nehmen. Die $K S M$ hat in den letzten Jahren und im Zuge der unterschiedlichen Aushandlungsprozesse über die behördliche und sozialpädagogische Zuständigkeit zunehmend deutlicher Positionen vertreten, die an den Grundsätzen der Kinder- und Jugendhilfe orientiert sind. Sie betont u. a., dass vertrauensvolle Arbeitsbeziehungen und ein Beachten des Datenschutzes notwendig seien; ein verfrühtes Intervenieren von Sicherheitsbehörden sei demgegenüber zu vermeiden. Für sie wäre eine anlassbezogene Kooperation in konkreten Fällen ausreichend. Es gab über mehrere Jahre eine Auseinandersetzung darum, bei welchem Ministerium eine ressortübergreifende Koordinierungsstruktur zur regionalen Bearbeitung von demokratiefeindlichem Islamismus anzusiedeln sei. Die SIM hatte ein großes Interesse an der Etablierung einer solchen umfassenden Netzwerkstruktur und sie entsprechend initiiert. Aus Sorge vor Vorbehalten im Falle einer Ansiedlung bei der Innenbehörde war es ihr Anliegen, der $K S M$ die „Schirmherrschaft“ (SIM, Z. 491) zu übertragen, was diese jedoch abgelehnt hat. So übernahm schließlich doch das Innenministerium diese Aufgabe.

Die zivilgesellschaftlichen Projekte Porto und Prag sind beide im Bereich der sozialpädagogischen Prävention tätig. Porto arbeitet in unterschiedlichen Modulen im Bereich von universeller bis indizierter Prävention, zum Teil auch in der Ausstiegsarbeit (s. unten) und hat dadurch in verschiedenen Konstellationen mit sicherheitsbehördlichen Akteur*innen zu tun. Das Projekt orientiert sich zentral an fachlichen Grundsätzen der Sozialen Arbeit und relativiert die Dringlichkeit des sozialen Problems zumindest im Hinblick auf die wachsende Bearbeitung durch Sicherheitsbehörden. Es verweist darauf, dass durch neu geschaffene Stellen auf Seiten der Sicherheitsbehörden die Gefahr bestehe, dass Bedarfe dadurch erst geschaffen werden bzw. Probleme größer erscheinen als sie es eigentlich sind. Vor diesem Hintergrund forcieren auch sie eine anlassbezogene Kooperation in konkreten Fällen statt umfassenderer Strukturen. Das Projekt Prag ist im universalpräventiven Bereich tätig und arbeitet vor allem mit Fachkräften sowie muslimisch sozialisierten jungen Menschen. Mit Sicherheitsbehörden haben sie in ihrer konkreten Arbeit wenig direkte Berührungspunkte. Der Träger ist gegenüber einer institutionalisierten Kooperation mit Sicherheitsbehörden eher kritisch eingestellt, weil er um das Vertrauen für die eigene Arbeit vor allem in muslimischen Communities fürchtet.

Im Hinblick auf die Problemdeutungen zeigen sich vor allem im Umgang mit der Kategorie des Gefährders ${ }^{9}$ relevante Unterschiede. Die SIM arbeitet mit den Kategorien Gefährder bzw. gefährliche Person ${ }^{10}$ zur Kategorisierung von Personen, „die Straftaten von einer Intensität begehen oder planen, die eine Gefährdung der

\footnotetext{
9 „Gefährder“ ist ein Arbeitsbegriff der Polizei, der „Personen benennen und identifizieren soll, von denen in Zukunft möglicherweise eine terroristische Gefahr ausgehen könnte" (Kretschmann und Legnaro 2019, S. 16).

${ }^{10}$ Polizei und Verfassungsschutz nutzen hier jeweils unterschiedliche Kategorien, wobei davon auszugehen ist, dass es grundsätzlich Überschneidungen gibt.
} 
inneren Sicherheit Deutschlands darstellt“ (SIM, Z. 197). Damit geht die Vorstellung einher, dass Gefährderkonzepte mit Blick auf konkrete Personen klar bestimmbar sind. Nach aktueller Rechtslage können Gefährder ohne deutsche Staatsbürgerschaft abgeschoben werden. Der interviewte Mitarbeiter äußert zwar inhaltliche Zweifel an dieser Praxis, verweist aber auf den Sachverhalt, dass dies nun aktuell die politische Lage sei. ${ }^{11}$ Die KSM sieht das Konzept des Gefährders kritisch und verweist auf aus ihrer Sicht ungeklärte Fragen bei der Einstufung einzelner Personen, etwa nach welchen Faktoren jemand als gefährdend eingestuft wird. Auch die zivilgesellschaftlichen Projekte sehen das Konzept des Gefährders kritisch, bzw. im Fall von Porto, als unvereinbar mit den Grundsätzen Sozialer Arbeit. Gerade der sozialpädagogische, verstehende Zugang zu Adressat*innen verhindere die Übernahme derart simplifizierender Kategorisierungen in die eigene Handlungspraxis. Verschärfend kommt der Aspekt der Vorfeld-Orientierung und der damit verbundenen Prognose hinzu, da Gefährder oft nicht straffällig geworden sind, ihnen aber ein besonders hohes Risiko zugeschrieben wird, in Zukunft Straftaten zu begehen. Die Innenbehörden sind hier insofern die mächtigeren Akteure, als sie solche Kategorien setzen können. Das Projekt Prag beispielsweise verortet sich selbst im Bereich der Universalprävention und arbeitete nicht mit Fällen mit Sicherheitsbezug. Sie beschreiben ihren Arbeitsbereich wie folgt: „Wir arbeiten ja nicht in dem Sinne mit Gefährdern“ (Prag, Z. 1131). Zwar lehnen sie diese Kategorisierung von Personen prinzipiell ab, verwenden sie jedoch zur Abgrenzung ihres Arbeitsbereichs.

In Bezug auf die Frage nach möglichen Tendenzen einer Versicherheitlichung des Arbeitsfeldes zeigt der Blick in die empirischen Daten, dass die Kategorie des Gefährders, auch wenn sie von den eher sozialpädagogisch verorteten Akteuren abgelehnt wird, zumindest im Sprechen und der Charakterisierung von Arbeitsbereichen ein zentraler Begriff ist und damit strukturierendes Gewicht erhält. Auf diese Weise spiegeln sich sicherheitsbehördliche Kategorien und ihnen inhärente Logiken von gesellschaftlichen Risiken und Gefahren letztlich auch in sozialpädagogischen Kontexten wider. Gerade, weil kooperative Aushandlungen gemeinsame Begriffe voraussetzen, kann im Durchsetzen von Begriffen und Konzepten ein Indiz für spezifische Machtkonstellation gesehen werden. So verdeutlicht dieses Beispiel eine ungleich verteilte Diskursmacht: Die sicherheitsbehördlichen Akteur*innen können diesen Begriff setzen und er findet im Ergebnis Eingang in den Sprachgebrauch der sozialpädagogischen Kooperationspartner*innen.

\section{Aushandlung der Zuständigkeit für Ausstiegsarbeit}

Das Projekt Porto war für mehrere Jahre für die Ausstiegsarbeit zuständig, sowohl direkt mit jungen Menschen als auch indirekt über die Beratung und Begleitung des

\footnotetext{
11 Besonders in dem Interview mit der SIM findet sich in einigen Passagen eine Distanzierung des Mitarbeitenden von der eigenen Hausleitung und ihren Positionen; etwa bei der politischen Forderung nach der Abschiebung von Gefährdern. Auch wenn sich im restlichen Interview, ebenso wie bei den anderen Interviewten, ein starkes kollektives „Wir“ zeigt, ist es wichtig eine interne Differenzierung der Akteur*innen mit zu bedenken.
} 
sozialen Umfelds. ${ }^{12}$ Die Abschiebung eines jungen Menschen mit Gefährder-Status, der in sozialpädagogischer Begleitung war, wird als Schüsselereignis genannt, nach dem Porto die Begleitung in ähnlichen sicherheitsrelevanten Fällen zurückweist. Das Projekt lehnt eine Konstellation ab, in der es von der SIM Arbeitsaufträge erhält und als sozialpädagogischer Träger mit Personen arbeiten soll, die von sicherheitsbehördlicher Seite als gefährlich eingestuft wurden. Aus seiner Sicht handelt es sich dabei um eine Instrumentalisierung Sozialer Arbeit. Sicherheitsbehörden sind mit ihren Vorgehensweisen nicht in der Lage, selbst mit diesen Personen sozialpädagogisch zu arbeiten und richten daher Arbeitsaufträge, z. T. verbunden mit der Aufforderung zur Informationsweitergabe, an Akteur*innen Sozialer Arbeit.

Porto beruft sich auf Prinzipien Sozialer Arbeit und beklagt, dass beispielsweise in Austauschrunden in der Vergangenheit mitunter die Einschränkung des Freiwilligkeitsgebots von Sozialer Arbeit diskutiert wurde, was für sie eine zentrale Arbeitsgrundlage darstellt. Vor dem Hintergrund des Auftrags innere Sicherheit zu gewährleisten strebt die SIM tendenziell eine lückenlose Bearbeitung an und hat den Anspruch, dass mit allen Personen direkt sozialpädagogisch gearbeitet werden muss. Demgegenüber verweist Porto darauf, dass die Bearbeitungsmöglichkeiten, wie z.B. die freiwillige Inanspruchnahme eines Angebots als Grundlage für ein beziehungsbasiertes Arbeitsbündnis, oft eingeschränkt sind, was die Sicherheitsbehörden jedoch kaum beachten würden. Darüber hinaus würden diese Fälle über eine Arbeit mit dem familiären Umfeld bereits abgedeckt. Zum Interviewzeitpunkt war die SIM daraufhin in Verhandlung mit einem anderen zivilgesellschaftlichen Träger, der ebenfalls in diesem Handlungsfeld aber in einem anderen Bundesland aktiv ist. Die SIM wird in Zukunft nun, als Ergebnis der erfolgten Grenzziehung, in der indizierten Prävention bzw. der Ausstiegsarbeit mit zwei unterschiedlichen zivilgesellschaftlichen Träger zusammenarbeiten: mit Porto in Fällen ohne Sicherheitsbezug und mit dem neuen Träger in Fällen mit möglichem Sicherheitsbezug. Mit der Einbeziehung eines zweiten Trägers demonstriert die SIM ihre Durchsetzungs- und Entscheidungsmacht in dieser regionalen Konstellation.

Der Widerstand in Form der Ablehnung von direkten sicherheitsbezogenen Arbeitsaufträgen zeigt das Vermögen des sozialpädagogischen Akteurs Porto, hier aktiv im Kampf um professionelle Legitimität zu agieren, fachliche Außengrenzen zu ziehen und so den Kooperationsanforderungen der SIM entgegen zu wirken. Dies wird in der konkreten Situation u. a. dadurch begünstigt, dass das Projekt im Handlungsfeld langjährig etabliert, vernetzt und erfahren in der fachlichen Positionierung ist. Darüber hinaus hat es mit der KSM einen behördlichen Akteur an der Seite, der ähnliche professionelle Positionen und Grenzziehungen vertritt. Mit Blick auf die positive Einschätzung von Kurt Möller und Florian Neuscheler (2018), intermediäre Strukturen in die Aushandlungen mit Sicherheitsbehörden zu etablieren, lassen sich hier ähnliche entlastende Potenziale in der Kooperation von Porto mit der KSM erkennen.

\footnotetext{
12 In der Ausstiegsarbeit wird im Handlungsfeld des demokratiefeindlichen Islamismus vergleichsweise häufig mit dem sozialen Umfeld, meist den Eltern, gearbeitet.
} 


\section{Positionierungen im regionalen Bearbeitungsarrangement}

Für eine erste Zusammenfassung sowie eine Zuspitzung zu klareren Rollenbeschreibungen im regionalen Kooperationsarrangement dient Abb. 1. Die eckig dargestellten Akteur*innen agieren in der politischen Arena, während die oval dargestellten Akteur*innen zivilgesellschaftliche Träger sind. Zugleich zeigt die Farbe die professionelle Verortung der Akteur*innen an - blau markiert ihren sozialpädagogischen Bezug und lila eine starke Sicherheitsorientierung. Abb. 1 stellt die Akteure in ihrer wechselseitigen Bezogenheit dar: Während die SIM kooperationsfordernd auftritt, relativiert die KSM die Bearbeitungsdringlichkeit und verweist auf fachliche sozialpädagogische Grenzen in der Kooperation. Die beiden zivilgesellschaftlichen Projekte reagieren auf die kooperationsoptimistischen Forderungen der SIM einerseits fachlich widerständig und andererseits mahnend. Die KSM gibt dabei den beiden zivilgesellschaftlichen Trägern fachliche Rückdeckung.

In der folgenden tiefergehenden Analyse betrachten wir die Aushandlungen in dem lokalen Kooperationsarrangement. Fokussiert wird in der weiteren Darstellung auf die beiden Akteure SIM und Porto, da sie besonders zentral für die Beschreibung des Verhältnisses zwischen sicherheitsbehördlichen und sozialpädagogischen Akteuren sind. ${ }^{13}$ Auf Basis der Interpretation von Schlüsselstellen arbeiten wir die forcierten Sicherheitsinteressen und den Umgang mit diesen heraus. Dabei verdichten wir die Positionierungen der einzelnen Akteur*innen im Verhältnis zu den jeweils anderen.

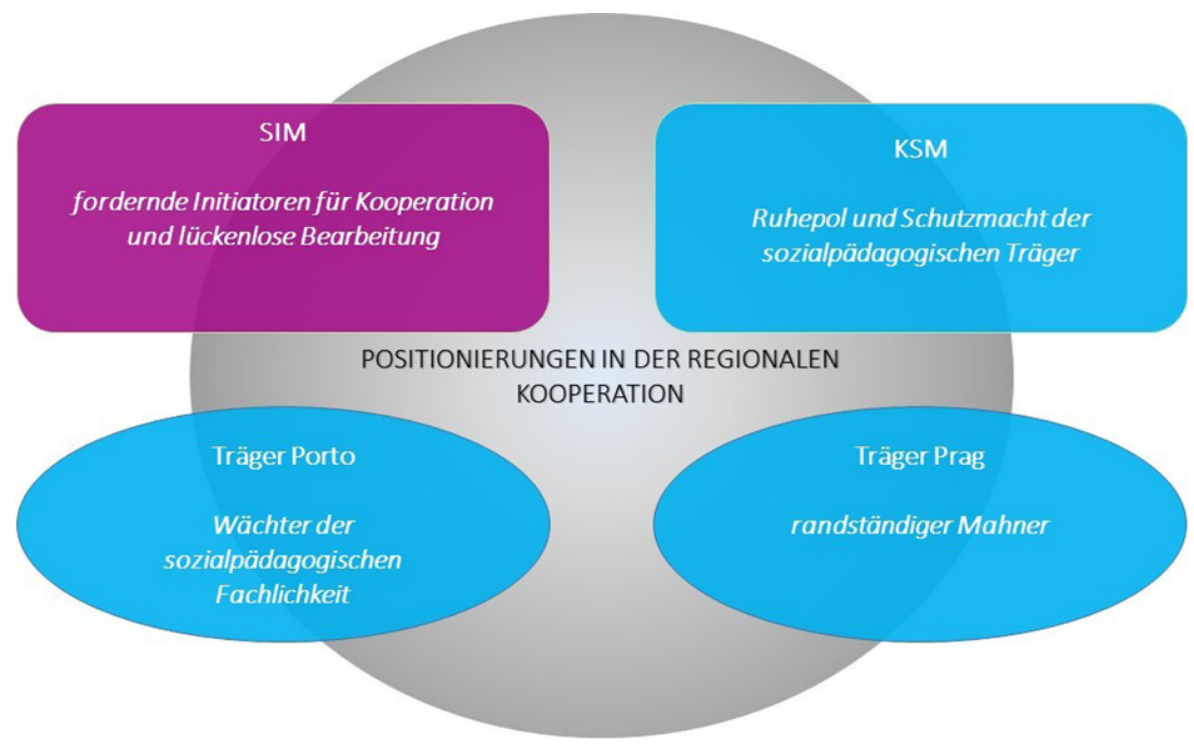

Abb. 1 Akteur*innen und ihre Positionierungen im regionalen Kooperationsarrangement

${ }^{13}$ Auch die anderen beiden Träger prägen die regionale Kooperationskonstellation. Aber sie sind zum einen als Sozialbehörde und zum anderen als Träger universalpräventiver Angebote weniger in die direkten Aushandlungen um Sicherheitskooperationen eingebunden. 


\subsection{SIM - Fordernde Initiatoren für Kooperation und lückenlose Bearbeitung}

Die SIM ist als Institution die treibende Kraft in der kooperativen Bearbeitung von sicherheitsrelevanten Fragen im Bereich des demokratiefeindlichen Islamismus. Sie unterstellt den anderen Kooperationspartnern ein geteiltes Interesse an entsprechender Informationsgewinnung und erkennt einen Konflikt um die Möglichkeiten der Informationsweitergabe:

Ja, klar, also die Sicherheitslage ist Ausgangspunkt aller Überlegung, und im Endeffekt ist dort der Druck insoweit auf jedes Ressort hoch, dass man ja nicht dort sitzen will und sagen kann, man hat nichts anzubieten. [...] Fakt ist, es hat Radikalisierungsprozesse im Bundesland $\mathrm{X}$ gegeben, das ist auch alles bekannt, und insofern kann sich auch da niemand wirklich rausreden. Nächstes Thema ist Datenschutz [...] das ist eine der allergrößten Herausforderungen in diesem gesamten Themenfeld: $\mathrm{Ab}$ wann kann überhaupt etwas übermittelt werden? Wenn man sich die Gesetzeslage anschaut, ist das ja eigentlich alles völlig klar. Sozialgesetzbuch und dort steht das abstrakt drin. Aber natürlich, wenn man eine Prozessbegleitung will, möchte man ja nicht erst erfahren, was passiert, wenn das Kind schon in den Brunnen gefallen ist, $d *$ ie Sozialarbeiter aber wiederum natürlich sagt, er macht sich ja strafbar, wenn er auch nur in irgendeiner Weise Informationen preisgibt über einen Prozess, selbst wenn er positiv verläuft. (SIM, Z. 802-829)

Im ersten Satz zeigt sich, dass hier die innere Sicherheit zur zentralen Denkfigur wird. Dass innere Sicherheit und damit der Schutz der demokratischen Verfassung und politischen Organe „Ausgangspunkt aller Überlegungen“ sei, verdeutlicht den verinnerlichen Arbeitsauftrag. Sie ist systemimmanent unausweichlich und hat oberste Priorität. Aus dieser Perspektive werden gesamtgesellschaftliche Sicherheitsinteressen und die Wahrung des politischen Systems auch zum Bestandteil von ressortübergreifenden Abwägungen. Zugleich wird von der "Sicherheitslage“ im Singular gesprochen und nicht auf Konstruktions- und Aushandlungsprozesse verwiesen. Dies stützt die Annahme, dass es sich dabei aus Sicht der SIM um eine objektive Lagebeschreibung handeln könne. Diese Objektivitätsvorstellung deuten wir auch als Ausdruck von kartierend-klassifizierendem Wissen (vgl. Schuhmacher 2018b, S. 121; Schiffauer 2015), das Eindeutigkeiten produzieren möchte und mit der Vorstellung einer objektivierbaren Einschätzung von Gefährdern einhergeht (Abschn. 4). Reflexionen auch über die Einschätzbarkeit der Sicherheitsrelevanz scheinen hier keine Rolle zu spielen, ebenso wie Differenzen in der Einschätzung der Sicherheitslage.

Die SIM erwartet vor dem Hintergrund auch von den anderen institutionellen Akteur*innen eine ressortübergreifende Kooperation. In der passiven Formulierung „der Druck [ist] insoweit auf jedes Ressort hoch, dass man ja nicht dort sitzen will und sagen kann, man hat nichts anzubieten" wird (zunächst) vermieden, dass die SIM selbst fordernd agiert. Andererseits liegt dem ressort- und professionsübergreifenden Kooperationsinteresse auch eine Interessensangleichung zugrunde, die davon ausgeht, dass alle beteiligten Fachkräfte zur möglichst lückenlosen Informationslage beitragen wollen. Vor dem Hintergrund des aktuellen Drucks in dem 
Handlungsfeld - welcher u. a. politisch generiert wird und an die SIM besonders hohe Sicherheitserwartungen stellt - löst die interviewte Person dies einseitig in Richtung Wissensmaximierung als Handlungsmaxime auf. Spätesten an der Stelle „da [kann sich] niemand wirklich rausreden“ wird deutlich, dass hier im Kooperationsarrangement auch eine Machtposition seitens der SIM beansprucht wird. Die $S I M$ will (wie vorherige Passagen zeigen) ihr eigenes Vertrauensdefizit in der Öffentlichkeit und in spezifischen Milieus kompensieren und ihre Informationsdichte durch die Kooperation ausbauen. Implizit wird damit seitens der Sicherheitsbehörden eine spezifische Qualität und Leistung der Sozialen Arbeit anerkannt, nämlich den Aufbau von vertrauensvolle Klient*innenbeziehungen, die für die Sicherheitsbehörden nutzbar gemacht werden sollen. Zugleich besteht auch ein Bewusstsein für rechtliche Grenzen. Durch die Einlassung, dass diese Begrenzung abstrakt klar sei, wird ein angenommener Verhandlungsspielraum im Detail bzw. gegebenenfalls ein Verhandlungsinteresse in konkreten datenschutzrechtlichen Fragen markiert. Damit setzt die SIM die innere Sicherheit gemäß ihrem Arbeitsauftrag absolut und nimmt sie nicht nur zum Ausgangspunkt der Überlegungen, sondern auch zum Endpunkt aller Strategien und Handlungen. Entwickelt man die Gegenperspektive zur Tendenz der Versicherheitlichung der Sozialen Arbeit, kann gefragt werden, ob die SIM nicht auch (verständlicherweise) gegen eine Sozialpädagogisierung ihres Arbeitsfeldes an arbeitet, d.h. gegen einen Prozess hin zur primären Orientierung an sozialpädagogischen Hilfe- und Unterstützungslogiken - auch in sicherheitsbezogenen Risikokonstellation.

Zugleich finden sich in dem Interview an vielen Stellen Formulierungen, in denen der Mitarbeitende der SIM Verständnis für die Differenzen zur anderen Handlungslogik und den Prinzipien der Sozialen Arbeit äußert. Vielfach entfaltet sich hier ein analytischer Blick auf unterschiedliche professionelle Systeme. Aber in den konkreten Kooperationsarrangements werden reibungsvolle Auseinandersetzungen normalisiert und die eigenen Forderungen als professionelle Notwendigkeiten begründet. Auch wenn dem sicherheitsbehördlichen Akteur bewusst ist, dass sie als Institution vielfach keinen rechtlichen Anspruch auf die Informationsweitergabe haben, so sind sie doch automatisch zuständig, wenn Fälle Sicherheitsrelevanz aufweisen.

\subsection{Porto - Wächter der sozialpädagogischen Fachlichkeit}

Der sozialpädagogische Träger Porto ist skeptisch gegenüber der als machtvoll wahrgenommen SIM und fordert in der Arbeit, auch mit sicherheitsrelevanten Fällen, eine fachliche Orientierung an Prinzipien der Sozialen Arbeit. Auch wenn sich der Träger gezielt aus der Zuständigkeit und Zusammenarbeitserwartung in sicherheitsrelevanten Fällen zurückgezogen hat (vgl. Abschn. 5), will er Wächter der Fachlichkeit bleiben.

Und jetzt kommen halt mehrere neue Akteure dazu [...] und da versuchen wir eigentlich trotzdem irgendwie so eine Klammer beizubehalten. [...] Wir müssen halt gucken, dass es irgendwie fachlich bleibt und fachlich vorangeht, dass wir so eine Linie beibehalten können, dass wir, also dass es ein gemeinsames Verständnis gibt im Umgang mit dem Phänomen, und vor allem, sozusagen auch 
an der Lebenswelt und an der Realität junger Menschen ausgerichtet, oder an der Menschen, die den Bedarf haben. Und das ist halt, ich sehe da immer noch die Soziale Arbeit mit Fachlichkeit und Professionalität am meisten gefragt so, und die sollte eigentlich auch sozusagen die Leitlinie eigentlich bilden, so eine Haltung. Aber das Problem ist, das Verständnis haben halt Menschen nicht, die beim Innenministerium haben häufig. Also, das ist kein Vorwurf, das müssen die auch nicht haben. Aber, nur ist halt das Problem, wenn die dann in einen Bereich einsteigen, wo es eigentlich genau dieses Verständnis braucht, wo man nicht vereinfachend von „Gefährdung“ ausgehen kann und vereinfachend von irgendwie bestimmten Charakteristika, die dazu führen, dass jemand gefährlich ist, dann ist es ein bisschen schwierig, weil wir da, weil die Akteure halt doch sehr viel Macht haben. [Porto, Z. 1108-1135]

In der Passage wird deutlich, dass der Mitarbeitende von Porto selbstverständlich die Debatten um die Fachlichkeit im Bereich der Radikalisierungsprävention beeinflussen will. Aufgrund seiner langjährigen Erfahrung in dieser Präventions- und Ausstiegsarbeit sieht das Projekt auch Soziale Arbeit als vorrangig zuständig für die Arbeit mit Adressat*innen an. Dies erzeugt insofern eine überaus ambivalente Situation, als dass sie für die Akzeptanz der professionellen Handlungslogik und Haltung der Sozialen Arbeit kämpfen, aber als Träger die professionelle Autonomie voranstellen und unter Bedingungen des landespolitischen Drucks der forcierten Datenweitergabe die trägereigene Zuständigkeit zurückweisen. Im Versuch fachliche Standards „,beizubehalten“ drückt sich aus, dass es diesen fachlichen Konsens im Kooperationsarrangement bereits in der Vergangenheit gab und auch in der direkten Einzelarbeit mit sicherheitsrelevanten Fällen nicht dahinter zurückgegangen werden soll. Aber die Aussage ,[w]ir müssen halt gucken“ verweist darauf, dass Porto sich des offenen Ausgangs der Aushandlungen bewusst ist. So soll in ihrer Vorstellung auch der neue Träger in Zukunft an den Lebensrealitäten und Bedarfen von jungen Menschen ausgerichtet sein und sich damit grundlegend auf die Prinzipien der lebensweltlichen und bedarfsorientierten Sozialen Arbeit berufen. In dieser Forderung manifestiert sich (zumindest in der beobachteten Etablierungs- und Übergangsphase) ihr Selbstverständnis in der Interaktionskonstellation als Wächter der sozialpädagogischen Fachlichkeit. Sie geben vor, was sie als gute Praxis in der Arbeit mit sicherheitsrelevanten Adressat*innen verstehen und rücken von ihrem fachlichen Maßstab auch in diesem Bereich nicht ab, ,wo es eigentlich genau dieses Verständnis braucht"“.

Die eigene Fachlichkeitswahrnehmung lebt vom Kontrast v. a. zu den sicherheitsbehördlichen Institutionen. Aus Sicht von Porto steigt die SIM nun mit viel Geld in das Arbeitsfeld ein. Dabei sieht die interviewte Person, dass es keinen gemeinsamen fachlichen Konsens zwischen Porto und der SIM geben kann, denn in der Fall- und Risikoeinschätzung gehen beide sehr unterschiedlich vor. Die SIM - so der fachlich und auch ethische Vorwurf - wolle mit vereinfachten Instrumenten nur den Fall eindeutig klassifizieren, während sozialpädagogische Akteur*innen den Fall in ihrer Komplexität verstehen und an den (dahinterliegenden) Bedarfen ansetzen wollen. Hier nehmen sie professionelle Differenzen und Wissenskulturen wahr, indem sie eine kartierend-klassifizierende Wissenskultur der Sicherheitsbehörden und 
ein eher hermeneutisch-prozessuales Wissen (Schiffauer 2015) der Sozialen Arbeit unvereinbar gegenüberstellen.

Auch wenn sie im gesamten Interview den Sicherheitsbehörden mit Verständnis für deren Auftrag begegnen, zeigt sich zugleich, dass Porto die eigenen fachlichen Standards der Sozialen Arbeit absolut setzt. Denkt man etwa die Idee der gemeinsamen fachlichen Klammer weiter, müsste es im Interesse von Porto sein, auch unter den sozialpädagogischen Akteur*innen die Normen der Selbstermächtigung der Adressat*innen, der Bedarfsorientierung und des verstehenden Zugangs durchzusetzen. So könnten die sozialpädagogischen Träger gerade in der sicherheitsdominierten Kooperation - wie Porto die Konstellation wahrnimmt - diese Position fachlich geschlossen nach außen vertreten und im Anschluss an Franz Josef Krafeld (s. oben) die ordnungspolitische Inanspruchnahme Sozialer Arbeit zurückweisen. Porto scheint die teils auch kämpferischen Rolle als Wächter der sozialpädagogischen Fachlichkeit verinnerlicht zu haben und demonstriert das eigenständige Profil von Sozialer Arbeit offensiv nach außen, um so einer möglichen Versicherheitlichung zu begegnen.

\section{Fazit}

Bezogen auf die eingangs formulierte Frage nach der Versicherheitlichung der sozialpädagogischen Bearbeitung von demokratiefeindlichem Islamismus zeigt sich in der explorativen Fallstudie, dass Sicherheitslogiken in dem betrachteten multiprofessionellen Kooperationsarrangement eine wichtige Rolle einnehmen. Vor allem wird deutlich, dass sicherheitsbehördliche Akteur*innen hier ein hohes Kooperationsinteresse haben und Anforderungen an sozialpädagogische Akteur*innen stellen, etwa in Bezug auf den Austausch von Informationen. Die Ergebnisse der Analyse verweisen darauf, dass Sicherheitsbehörden bzw. Innenministerien in diesem regionalen, multiprofessionellen Arrangement zwar nicht gänzlich dominieren, zugleich wurden ungleiche Machtverhältnisse sichtbar. Insbesondere die sozialpädagogischen Träger nehmen sicherheitsbehördliche Akteur*innen als ressourcenstark und aushandlungsmächtig wahr. Das Beispiel der Kategorie „Gefährder“, die unter der Logik von Gefahrenabwehr von sicherheitsbehördlichen Akteur*innen etabliert wurde, verdeutlicht dabei die ungleich verteilte Diskursmacht. Zwar lehnen die drei sozialpädagogischen Akteur*innen das Konzept und das dahinterstehende Fallverständnis ab, dennoch verwenden sie den Begriff und tragen damit zur Etablierung des Konzepts bei.

Der Versuch, zunehmend sicherheitspolitisch gerahmte multiprofessionelle Strukturen zu etablieren, kann als Hinweis darauf angesehen werden, dass sich Tendenzen einer Versicherheitlichung des Handlungsfeldes abzeichnen. Die vorliegende empirische Fallanalyse legt den Schluss nahe, dass sicherheitsbehördliche Akteur*innen tendenziell die unterschiedlichen professionsbezogenen Wissenskulturen in Richtung einer sicherheitsbezogenen Wissensmaximierung auflösen wollen. Dabei handelt es sich in dem betrachteten Kooperationsarrangement jedoch lediglich um Versuche, Sicherheitsinteressen durchzusetzen. Es würde zu weit führen, hier von versicherheitlichten Kooperationsarrangements im Ergebnis zu sprechen, da diese Ansin- 
nen von den sozialpädagogischen Akteur*innen zum Teil zurückgewiesen werden. Sie widersetzen sich und ziehen professionsbezogene Zuständigkeitsgrenzen: So lehnt etwa ein zivilgesellschaftlicher Träger den Arbeitsauftrag der sozialpädagogischen Ausstiegsarbeit mit Personen, die als Gefährder kategorisiert sind, ab. Hier erfolgt eine klare Grenzziehung unter Rückgriff auf Fachlichkeitsprinzipien der eigenen Profession. In diesem Fall ermöglicht eine Profilschärfung auch in tendenziell asymmetrischen Machtkonstellationen fachlich begründete Grenzziehungen.

Inwieweit sich Sicherheitsinteressen in der sozialpädagogischen Bearbeitung von demokratiefeindlichem Islamismus durchsetzen, hängt nicht zuletzt von regionalen bzw. lokalen Kontexten und Akteur*innen ab. So zeigt unsere Fallstudie durch die Rekonstruktion von Prozessen des doing cooperation, dass eine fachlich begründete Grenzziehung, hier in Form eines Rückzugs, voraussetzungsvoll, aber möglich ist. Es braucht dafür Vernetzung unter den sozialpädagogischen Akteur*innen, ein Bewusstsein für professions- und trägereigene fachliche Prinzipien und Erfahrungen, sowohl in der Arbeit als auch in der (gemeinsamen) fachlichen Positionierung. Aber auch die strukturellen Rahmenbedingungen der Trägerförderung sind nicht unerheblich, denn diese müssen es einzelnen Trägern erlauben, im Zweifelsfall auf öffentliche Gelder zu verzichten. Analog zu den Beobachtungen von Bernd Dollinger (2014) für Soziale Arbeit im Allgemeinen sind die Entwicklungen in Richtung einer Dominanz sicherheitspolitischer Wissensmaximierung und Kooperationsstrukturen auch im hier betrachteten Fall zwar nicht umfassend, aber dennoch ernst zu nehmen (Dollinger 2014, S. 309). So setzt sich beispielsweise die sicherheitsbehördliche Anforderung, auch mit sicherheitsrelevanten Fällen sozialpädagogisch zu arbeiten trotz des Rückzugs des zivilgesellschaftlichen Projekts durch, denn es wird ein neuer sozialpädagogischer Akteur für diese Arbeit hinzugezogen. Dies kann auch als Plädoyer für einen differenzierteren Blick verwendet werden, weil hier nicht pauschal die sozialpädagogischen Akteur*innen den sicherheitsbehördlichen Akteur*innen als zwei homogene Lager gegenüberstehen.

Aus Perspektive „klassischer“ Arbeitsbereiche mag die indizierte Prävention bzw. Ausstiegsarbeit als Grenzfall Sozialer Arbeit anmuten, in dem Aspekte von Kontrolle dominieren. Diese Zuschreibung würde unseres Erachtens jedoch zu kurz greifen. Der bewusste Anschluss an Debatten Sozialer Arbeit stärkt angesichts einer sich abzeichnenden zunehmenden sicherheitspolitischen Rahmungen des Feldes die professionsbezogenen Positionierungen sozialpädagogischer Akteur*innen (vgl. für den Bereich Rechtsextremismus Huber und Rieker 2015, S. 83 f.). In Deutschland existieren bisher je nach Bundesland unterschiedliche Formen der Kooperation und Informationsweitergabe zwischen sozialpädagogischen und sicherheitsbehördlichen Akteur*innen. In dieser explorativen Fallastudie wurde eine regionale Konstellation untersucht, bei einer Einordnung in darüberhinausgehende Feldbeobachtungen ist allerdings nicht davon auszugehen, dass eine derart starke fachliche Positionierung sozialpädagogischer Akteur*innen und eine Akzeptanz dieser professionellen Außengrenze der bundesweite Regelfall ist. Es scheint daher weiterhin geboten, Tendenzen einer möglichen Versicherheitlichung dieses Handlungsfeldes in Zukunft stärker als bisher durch Forschung kritisch zu begleiten und die damit verbundenen Konsequenzen für sozialpädagogische Praxis empirisch im Zeitverlauf zu betrachten. 
Funding Open Access funding enabled and organized by Projekt DEAL.

Open Access Dieser Artikel wird unter der Creative Commons Namensnennung 4.0 International Lizenz veröffentlicht, welche die Nutzung, Vervielfältigung, Bearbeitung, Verbreitung und Wiedergabe in jeglichem Medium und Format erlaubt, sofern Sie den/die ursprünglichen Autor(en) und die Quelle ordnungsgemäß nennen, einen Link zur Creative Commons Lizenz beifügen und angeben, ob Änderungen vorgenommen wurden.

Die in diesem Artikel enthaltenen Bilder und sonstiges Drittmaterial unterliegen ebenfalls der genannten Creative Commons Lizenz, sofern sich aus der Abbildungslegende nichts anderes ergibt. Sofern das betreffende Material nicht unter der genannten Creative Commons Lizenz steht und die betreffende Handlung nicht nach gesetzlichen Vorschriften erlaubt ist, ist für die oben aufgeführten Weiterverwendungen des Materials die Einwilligung des jeweiligen Rechteinhabers einzuholen.

Weitere Details zur Lizenz entnehmen Sie bitte der Lizenzinformation auf http://creativecommons.org/ licenses/by/4.0/deed.de.

\section{Literatur}

Abbott, A.D. (1988). The system of professions. An essay on the division on expert labor. Chicago: University of Chicago Press.

Baban, C. P. (2013). Der innenpolitische Sicherheitsdiskurs in Deutschland. Zur diskursiven Konstruktion des sicherheitspolitischen Wandels 2001-2009. Wiesbaden: Springer VS.

BMI (2017b). Nationales Präventionsprogramm gegen islamistischen Extremismus. https://www.bmi. bund.de/SharedDocs/downloads/DE/veroeffentlichungen/themen/sicherheit/praeventionsprogrammislamismus.html. Zugegriffen: 8. Juni 2019.

Bröckling, U. (2008). Vorbeugen ist besser... Zur Soziologie der Prävention. Behemoth. A Journal on Civilisation, 1, 38-48.

Bundesministerium des Innern (BMI) (2017a). Verfassungsschutzbericht 2017. Berlin: BMI.

Dollinger, B. (2014). Soziale Arbeit als Realisierung protektiver Sicherheitspolitiken. Zeitschrift für Sozialpädagogik, 12(3), 296-314.

Dollinger, B., Bock, K., Böllert, K., Braches-Chyrek, R., Heite, C., Kessl, F., Thole, W., \& Ziegler, H. (2017). Editorial „Sicherheit“. Soziale Passagen, 9(2), 205-211.

Durodie, B. (2016). Securitising education to prevent terrorism or losing direction? British Journal of Educational Studies, 64(1), 21-35.

Franzheld, T. (2017). Profession und Kooperation - empirische Verhältnisse und theoretische Positionen. In Die herausgeforderte Profession. Soziale Arbeit in multiprofessionellen Handlungskontexten. neue praxis, Sonderheft 14. (S. 13-23).

Groenemeyer, A. (2013). Wege der Sicherheitsgesellschaft. Über den Zusammenhang von gesellschaftlicher Entwicklung, Politik und Sicherheitsmentalitäten im Alltag. In E. Marks \& W. Steffen (Hrsg.), Sicher leben in Stadt und Land. Ausgewählte Beiträge des 17. Deutschen Präventionstages (S. 177-194). Mönchengladbach: Forum Verlag Godesberg.

Handle, J., Korn, J., \& Mücke, T. (2020). Zivilgesellschaftliche Organisationen in der Tertiärprävention. https://violence-prevention-network.de/wp-content/uploads/2020/01/Violence-PreventionNetwork-Schriftenreihe-Heft-3-1.pdf. Zugegriffen: 4. Juli 2020.

Heinrich, B. (2017). Zum heutigen Zustand der Kriminalpolitik in Deutschland. KriPoZ, 1, 4-20.

Huber, S., \& Rieker, P. (2015). Der sozialpädagogische Umgang mit Devianz zwischen Hilfe und Kontrolle. In B. Dollinger \& N. Oelkers (Hrsg.), Sozialpädagogische Perspektiven auf Devianz (S. 68-86). Weinheim, Basel: Beltz Juventa.

Huber, S., \& Schierz, S. (2013). Punitivierung der Sozialen Arbeit? Anmerkungen zur gegenwärtigen Debatte. In P. Rieker, S. Huber, A. Schnitzer \& S. Brauchli (Hrsg.), Hilfe! Strafe! Multiperspektivische Reflexionen eines brisanten Spannungsverhältnisses (S. 102-118). Weinheim, Basel: Beltz Juventa.

Jukschat, N., \& Leimbach, K. (2019). Radikalisierung als hegemoniales Paradigma. Eine empiriebasierte kritische Bestandsaufnahme. BEHEMOTH A Journal on Civilisation, 12(2), 11-23.

Klatetzki, T. (2005). Professionelle Arbeit und kollegiale Organisation. Eine symbolisch interpretative Perspektive. In T. Klatetzki \& V. Tacke (Hrsg.), Organisation und Profession (S. 255-283). Wiesbaden: VS. 
Kretschmann, A., \& Legnaro, A. (2019). Abstrakte Gefährdungslagen. Zum Kontext der neuen Polizeigesetze. ApuZ, 21-23, 11-17.

Merten, U. (2016). Kooperation und Partizipation - ein ethisch gemischtes Doppel. In U. Merten \& P. Zängl (Hrsg.), Ethik und Moral in der Sozialen Arbeit - Wirkungsorientiert - kontextbezogen - habitusbildend (S. 187-212). Opladen: Barbara Budrich.

Möller, K. (2010). Soziale Arbeit und Polizei. Sozialarbeitswissenschaftlich-pädagogische Eckpunkte einer Zweckbeziehung. In K. Möller (Hrsg.), Dasselbe in grün? Aktuelle Perspektiven auf das Verhältnis von Polizei und Sozialer Arbeit (S. 14-27). Weinheim, München: Juventa.

Möller, K., \& Neuscheler, F. (2018). Abschlussbericht zur Evaluation der Beratungsstelle Hessen - Religiöse Toleranz statt Extremismus. https://violence-prevention-network.de/wp-content/uploads/2019/ 02/Abschlussbericht-Evaluation-Beratungsstelle-Hessen.pdf. Zugegriffen: 12. Juni 2019.

Müller, S. (2001). Erziehen - Helfen - Strafen. Das Spannungsverhältnis von Hilfe und Kontrolle in der Sozialen Arbeit. Weinheim, München: Juventa.

Nordbruch, G. (2018). Mehr als nur Dienstleister: Zivilgesellschaftliche Präventionsarbeit in Deutschland. In M. von Drachenfels, P. Offermann \& C. Wunderlich (Hrsg.), Radikalisierung und De-Radikalisierung in Deutschland. Eine gesamtgesellschaftliche Aufgabe (S. 93-99). Frankfurt am Main: Hessische Stiftung Friedens- und Konfliktforschung.

Said, B.T., \& Fouad, H. (2018). Countering Islamist radicalisation in Germany: a guide to Germany's growing prevention infrastructure. International Centre for Counter-Terrorism Policy Brief. https://icct.nl/wp-content/uploads/2018/09/ICCT-Said-Fouad-Countering-Islamist-Radicalizationin-Germany-Sept2018.pdf. Zugegriffen: 27. Mai 2019.

Schau, K., Langner, J., Glaser, M., \& Figlestahler, C. (2017). Demokratiefeindlichem und gewaltorientiertem Islamismus begegnen. Ein Überblick über Entwicklungen und Herausforderungen eines jungen pädagogischen Handlungsfeldes. In J. Kärgel (Hrsg.), „Sie haben keinen Plan B“ Radikalisierung, Ausreise, Rückkehr - zwischen Prävention und Intervention (S. 197-211). Bonn: Bundeszentrale für politische Bildung.

Scherr, A. (2014). Kriminalität, innere Sicherheit und soziale Unsicherheit. Sicherheitsdiskurse als Bearbeitung gesellschaftsstrukturell bedingter Ängste. Wiesbaden: Springer VS.

Schiffauer, W. (2015). Sicherheitswissen und Deradikalisierung. In Friedrich-Ebert-Stiftung (Hrsg.), Handlungsempfehlungen zur Auseinandersetzung mit islamistischem Extremismus und Islamfeindlichkeit. Arbeitsergebnisse eines Expertengremiums der Friedrich-Ebert-Stiftung (S. 216-243). Berlin: Friedrich-Ebert-Stiftung.

Schuhmacher, N. (2018a). Ein neues Bild der Prävention? Zur Tendenz der „Versicherheitlichung“ im pädagogischen Feld. In M. Glaser, A. Frank \& M. Herding (Hrsg.), Gewaltorientierter Islamismus im Jugendalter. Perspektiven aus Jugendforschung und Jugendhilfe (S. 158-166). Sozialmagazin, 2. Sonderband.

Schuhmacher, N. (2018b). Evaluation der Hamburger „Fach- und Beratungsstelle für religiös begründete Radikalisierung - Legato“. Universität Hamburg. https://www.wiso.uni-hamburg.de/fachbereichsowi/professuren/hentschel/forschung/legato/legato-abschlussbericht-voe.pdf. Zugegriffen: 15. Mai 2019.

Schütze, F. (1992). Soziale Arbeit als ,,bescheidene Profession“. In B. Dewe, W. Ferchhoff \& F. Olaf-Radtke (Hrsg.), Erziehen als Profession. Zur Logik professionellen Handelns in pädagogischen Feldern (S. 132-171). Opladen: Leske + Budrich.

Silkenbeumer, M., Thieme, N., \& Kunze, K. (2017). Kooperation in multiprofessionellen Handlungskontexten. Zur Frage beruflicher Zuständigkeit(en) Sozialer Arbeit. In Soziale Arbeit in multiprofessionellen Handlungskontexten. neue praxis, Sonderheft 14: Die herausgeforderte Profession. (S. 35-42).

Singelnstein, T., \& Stolle, P. (2012). Die Sicherheitsgesellschaft. Soziale Kontrolle im 21. Jahrhundert. Wiesbaden: VS.

Soeffner, H.-G. (2008). Sozialwissenschaftliche Hermeneutik. In U. Flick, E. von Kardorff \& I. Steinke (Hrsg.), Qualitative Forschung. Ein Handbuch (S. 164-175). Reinbek: Rowohlt.

Strauss, A. (1994). Grundlagen qualitativer Sozialforschung. Datenanalyse und Theoriebildung in der empirischen soziologischen Forschung. München: Fink. 